\title{
Electric and Magnetic Field Dyadic Green's Functions and Depolarizing Dyad for a Magnetic Current Immersed in a Uniaxial Dielectric-Filled Parallel Plate Waveguide
}

\author{
Michael Havrilla \\ 1'Air Force Institute of Technology, 2950 Hobson Way, WPAFB, OH 45434, USA, michael.havrilla@afit.edu
}

\begin{abstract}
The dyadic Green's functions and depolarizing dyad for the electric and magnetic fields due to a magnetic current immersed in a uniaxial dielectric-filled parallel plate waveguide with perfect electric conducting (PEC) boundaries are developed here. The Green's functions are derived directly from Maxwell's equations using the superposition of a particular (i.e., principal) and complementary (i.e., reflected) solution based upon a spectral domain analysis. It is shown that the magnetic current maintains both ordinary and extraordinary waves which are $T E^{z}$ and $T M^{z}$, respectively, and that these field sets do not couple at the parallel-plate PEC boundaries. The physical meaning of the various terms in the Green's functions is provided as well as a simple formulation for computing the magnetic field Green's function from the electric field Green's function. A simple approach for computing the depolarizing dyad term due to the source point singularity in the principal magnetic field representation is presented and consequently leads to a Green's function expression that is valid both externally and internally to the source region. Applications which motivate the need for these Green's functions, such as the measurement of hexagonal honeycomb core composites and uniaxial crystals, are also discussed.
\end{abstract}

\section{Introduction}

Electromagnetic material characterization is the process of determining the constitutive parameters (e.g., permittivity and permeability) of matter. Electromagnetic material characterization techniques can be broadly classified as destructive [1-2] and nondestructive [3-5]. In [5], for example, a simple (i.e., linear, homogeneous and isotropic) planar material is clamped between two flanged rectangular waveguides to form a parallel-plate region. It is shown there that the waveguide aperture regions may be replaced by equivalent magnetic currents immersed in the parallelplate region which allows theoretical expressions for reflection and transmission coefficients to be developed. These coefficients, when compared to measurement via a root search, lead to extracted permittivity and permeability values. In a similar manner and with appropriate Green's function development, uniaxial anisotropic dielectrics (such as crystals or hexagonal honeycomb core composites) may be characterized using this clamped flange technique (which is the future goal of this research). The objective of this paper is to derive the electric and magnetic field Green's functions due to a magnetic current immersed in a uniaxial dielectric-filled parallel-plate waveguide. The electric field dyadic Green's function is developed first via a spectral domain approach. Next, the magnetic field dyadic Green's function is computed from knowledge of electric field Green's function including a simple formulation which clearly identifies a depolarizing dyad term. Conclusions and future work are provided in the last section.

\section{Electric Field Dyadic Green's Function}

Consider a magnetic current $\vec{J}_{m}$ immersed in the parallel-plate waveguide with PEC boundaries at $z=0, d$ and filled with a uniaxial dielectric having permittivity $\vec{\varepsilon}=\hat{x} \varepsilon_{t} \hat{x}+\hat{y} \varepsilon_{t} \hat{y}+\hat{z} \varepsilon_{n} \hat{z}=\vec{I} \varepsilon_{t}+\hat{z} \hat{z}\left(\varepsilon_{n}-\varepsilon_{t}\right) \quad($ where $\vec{I}=\hat{x} \hat{x}+\hat{y} \hat{y}+\hat{z} \hat{z})$ as depicted below in Figure 1. A superposition of principal $(p)$ and reflected $(r)$ wave contributions is also illustrated.

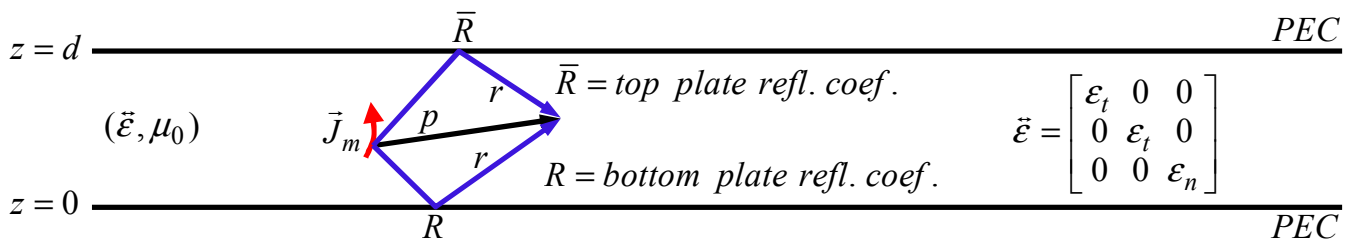

Figure 1: Parallel-plate waveguide filled with a uniaxial dielectric (principal axis aligned along $z-$ axis). 
Accordingly, Maxwell's equations for the principal (i.e., forced) and reflected (unforced) contributions are written as

$$
\begin{aligned}
& \vec{\nabla} \cdot \vec{E}^{p}(\vec{\rho}, z)=-\vec{J}_{m}(\vec{\rho}, z)-j \omega \mu_{0} \vec{H}^{p}(\vec{\rho}, z)_{\text {. . principal }}, \quad \vec{\nabla} \cdot \vec{E}^{r}(\vec{\rho}, z)=-j \omega \mu_{0} \vec{H}^{r}(\vec{\rho}, z) \ldots \text { reflected } \\
& \vec{\nabla} \cdot \vec{H}^{p}(\vec{\rho}, z)=j \omega \vec{\varepsilon} \cdot \vec{E}^{p}(\vec{\rho}, z) \quad \ldots \text { principal }, \quad \vec{\nabla} \cdot \vec{H}^{r}(\vec{\rho}, z)=j \omega \vec{\varepsilon} \cdot \vec{E}^{r}(\vec{\rho}, z)
\end{aligned}
$$

where the operator $\vec{\nabla}=\vec{I} \times \nabla, \nabla=\hat{x} \frac{\partial}{\partial x}+\hat{y} \frac{\partial}{\partial y}+\hat{z} \frac{\partial}{\partial z}$ and $\vec{\rho}=\hat{x} x+\hat{y} y$. Note, $\vec{\nabla} \cdot \vec{F}=\nabla \times \vec{F}$ for arbitrary vector $\vec{F}$. The parallel plates being infinite in extent along the $x$ and $y$ dimensions prompts Fourier transformation of (1), leading to

$$
\begin{aligned}
& \overrightarrow{\widetilde{\nabla}} \cdot \overrightarrow{\tilde{E}}^{p}\left(\vec{\lambda}_{\rho}, z\right)=-\overrightarrow{\tilde{J}}_{m}\left(\vec{\lambda}_{\rho}, z\right)-j \omega \mu_{0} \overrightarrow{\tilde{H}}^{p}\left(\vec{\lambda}_{\rho}, z\right) \quad \overrightarrow{\widetilde{\nabla}} \cdot \overrightarrow{\tilde{E}}^{r}\left(\vec{\lambda}_{\rho}, z\right)=-j \omega \mu_{0} \overrightarrow{\tilde{H}}^{r}\left(\vec{\lambda}_{\rho}, z\right) \\
& \overrightarrow{\widetilde{\nabla}} \cdot \overrightarrow{\tilde{H}}^{p}\left(\vec{\lambda}_{\rho}, z\right)=j \omega \vec{\varepsilon} \cdot \overrightarrow{\tilde{E}}^{p}\left(\vec{\lambda}_{\rho}, z\right) \quad, \quad \overrightarrow{\widetilde{\nabla}} \cdot \overrightarrow{\tilde{H}}^{r}\left(\vec{\lambda}_{\rho}, z\right)=j \omega \vec{\varepsilon} \cdot \overrightarrow{\tilde{E}}^{r}\left(\vec{\lambda}_{\rho}, z\right)
\end{aligned}
$$

where $\vec{\nabla}=\vec{I} \times \widetilde{\nabla}, \widetilde{\nabla}=j \vec{\lambda}_{\rho}+\hat{z} \frac{\partial}{\partial z}, \vec{\lambda}_{\rho}=\hat{x} \lambda_{x}+\hat{y} \lambda_{y}, \lambda_{\rho}^{2}=\vec{\lambda}_{\rho} \cdot \vec{\lambda}_{\rho}$ and the following generic transform pair has been used

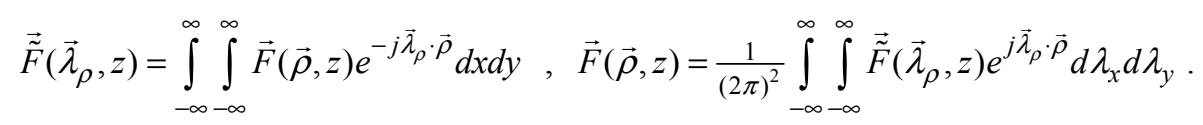

The principal component represents the field maintained by a magnetic current immersed in an unbounded uniaxial medium, thus further transformation on the $z$ variable of the principal field is prompted, producing the result

$$
\begin{aligned}
& \ddot{\widetilde{\nabla}} \cdot \overrightarrow{\tilde{E}}^{p}\left(\vec{\lambda}_{\rho}, \lambda_{z}\right)=j \vec{\lambda} \cdot \overrightarrow{\tilde{E}}^{p}\left(\vec{\lambda}_{\rho}, \lambda_{z}\right)=-\overrightarrow{\tilde{\tilde{J}}}_{m}\left(\vec{\lambda}_{\rho}, \lambda_{z}\right)-j \omega \mu_{0} \overrightarrow{\tilde{\tilde{H}}}^{p}\left(\vec{\lambda}_{\rho}, \lambda_{z}\right) \\
& \overrightarrow{\widetilde{\nabla}} \cdot \overrightarrow{\tilde{\tilde{H}}}^{p}\left(\vec{\lambda}_{\rho}, \lambda_{z}\right)=j \vec{\lambda} \cdot \overrightarrow{\tilde{\tilde{H}}}^{p}\left(\vec{\lambda}_{\rho}, \lambda_{z}\right)=j \omega \vec{\varepsilon} \cdot \overrightarrow{\tilde{\tilde{E}}}^{p}\left(\vec{\lambda}_{\rho}, \lambda_{z}\right)
\end{aligned}
$$

with $\overrightarrow{\widetilde{\nabla}}=\vec{I} \times \widetilde{\widetilde{\nabla}}=\vec{I} \times\left(j \vec{\lambda}_{\rho}+\hat{z} j \lambda_{z}\right)=j \vec{I} \times \vec{\lambda}=j \vec{\lambda}$ and the following generic $z-$ variable transform pair has been utilized

$$
\overrightarrow{\tilde{F}}\left(\vec{\lambda}_{\rho}, \lambda_{z}\right)=\int_{-\infty}^{\infty} \overrightarrow{\tilde{F}}\left(\vec{\lambda}_{\rho}, z\right) e^{-j \lambda_{z} z} d z \quad, \quad \overrightarrow{\tilde{F}}\left(\vec{\lambda}_{\rho}, z\right)=\frac{1}{2 \pi} \int_{-\infty}^{\infty} \overrightarrow{\tilde{F}}\left(\vec{\lambda}_{\rho}, \lambda_{z}\right) e^{j \lambda_{z} z} d \lambda_{z}
$$

Solving for $\overrightarrow{\tilde{H}}^{p}$ in Faraday's law and substitution into Ampere's law in (4) leads to the forced wave equation relation

$$
\vec{W}_{E} \cdot \overrightarrow{\tilde{\tilde{E}}}^{p}\left(\vec{\lambda}_{\rho}, \lambda_{z}\right)=-j \vec{\lambda} \cdot \overrightarrow{\tilde{\tilde{J}}}_{m}\left(\vec{\lambda}_{\rho}, \lambda_{z}\right) \Rightarrow \overrightarrow{\tilde{\tilde{E}}}^{p}\left(\vec{\lambda}_{\rho}, \lambda_{z}\right)=-j \overleftrightarrow{W}_{E}^{-1} \cdot \vec{\lambda}_{\overrightarrow{\tilde{J}}}\left(\vec{\lambda}_{\rho}, \lambda_{z}\right)=\overleftrightarrow{\widetilde{\widetilde{G}}}_{E H}^{p}\left(\vec{\lambda}_{\rho}, \lambda_{z}\right) \cdot \overrightarrow{\tilde{\tilde{J}}}_{m}\left(\vec{\lambda}_{\rho}, \lambda_{z}\right)
$$

where $\overleftrightarrow{W}_{E}=-\vec{\lambda} \cdot \vec{\lambda}-\overleftrightarrow{k^{2}}=\vec{I}\left(\lambda^{2}-k_{t}^{2}\right)-\vec{\lambda} \vec{\lambda}-\hat{z} \hat{z}\left(k_{n}^{2}-k_{t}^{2}\right), \quad k_{t}^{2}=\omega^{2} \varepsilon_{t} \mu_{0}, k_{n}^{2}=\omega^{2} \varepsilon_{n} \mu_{0}$ and $\stackrel{\widetilde{G}}{E H}_{E H}^{p}-j \overleftrightarrow{W}_{E}^{-1} \cdot \vec{\lambda}$ is the electric field dyadic Green's function in the $\vec{\lambda}$-domain. The term $\overleftrightarrow{W}_{E}^{-1}$ can be found via equation (1.128) in [6], thus

$$
\overleftrightarrow{\widetilde{G}}_{E H}^{p}\left(\vec{\lambda}_{\rho}, \lambda_{z}\right)=-j \overleftrightarrow{W}_{E}^{-1} \cdot \vec{\lambda}=-j \frac{\operatorname{adj} \overleftrightarrow{W}_{E}}{\operatorname{det} \vec{W}_{E}} \cdot \vec{\lambda}=-j \frac{\left(\lambda_{z}^{2}-\lambda_{z o}^{2}\right)\left[-\vec{I}_{n}^{2}+\hat{z} \hat{z}\left(k_{n}^{2}-k_{t}^{2}\right)\right]+(\vec{\lambda} \times \hat{z})(\vec{\lambda} \times \hat{z})\left(k_{n}^{2}-k_{t}^{2}\right)}{-k_{n}^{2}\left(\lambda_{z}^{2}-\lambda_{z o}^{2}\right)\left(\lambda_{z}^{2}-\lambda_{z e}^{2}\right)} \cdot \vec{\lambda}
$$

where $\lambda_{z o}=\left(k_{t}^{2}-\lambda_{\rho}^{2}\right)^{\frac{1}{2}}$ and $\lambda_{z e}=\frac{k_{t}}{k_{n}}\left(k_{t}^{2}-\lambda_{\rho}^{2}\right)^{\frac{1}{2}}$ are the $z$-directed propagation factors (i.e., pole singularities in the $\vec{\lambda}$-domain), respectively. Note, the relation $\vec{\lambda} \cdot \vec{\lambda}=0$ was implicitly used to obtain the result in (7). Fourier inversion leads to the $\vec{\lambda}_{\rho}$ - domain electric field representation (with generic current existing in the guide region $0 \leq z \leq d$ )

$$
\overrightarrow{\tilde{E}}^{p}\left(\vec{\lambda}_{\rho}, z\right)=\int_{0}^{d} \widetilde{\widetilde{G}}_{E H}^{p}\left(\vec{\lambda}_{\rho} ; z-z^{\prime}\right) \cdot \overrightarrow{\widetilde{J}}_{m}\left(\vec{\lambda}_{\rho}, z^{\prime}\right) d z^{\prime}, \overleftrightarrow{\widetilde{G}}_{E H}^{p}\left(\vec{\lambda}_{\rho} ; z-z^{\prime}\right)=\frac{1}{2 \pi} \int_{-\infty}^{\infty} \overleftrightarrow{\widetilde{G}}_{E H}^{p}\left(\vec{\lambda}_{\rho}, \lambda_{z}\right) e^{j \lambda_{z}\left(z-z^{\prime}\right)} d \lambda_{z} .
$$

Application of Cauchy's integral theorem in (8) along with (7) produces the desired principal Green's function result 


$$
\overrightarrow{\widetilde{G}}_{E H}^{p}=\overrightarrow{\widetilde{G}}_{E H}^{p o}+\overrightarrow{\widetilde{G}}_{E H}^{p e}=\frac{\left(\hat{\lambda}_{\rho} \times \hat{z}\right)\left(\hat{\lambda}_{\rho} \times \hat{z}\right) \cdot \vec{\lambda}_{o}}{-2 \lambda_{z o}} e^{-j \lambda_{z o}\left|z-z^{\prime}\right|}+\frac{\vec{\lambda}_{e}-\hat{z} \hat{z} \vec{\lambda}_{e}\left[1-\left(\frac{k_{t}}{k_{n}}\right)^{2}\right]-\left(\hat{\lambda}_{\rho} \times \hat{z}\right)\left(\hat{\lambda}_{\rho} \times \hat{z}\right) \cdot \vec{\lambda}_{e}}{-2 \lambda_{z e}} e^{-j \lambda_{z e}\left|z-z^{\prime}\right|}
$$

where $\hat{\lambda}_{\rho}=\frac{\vec{\lambda}_{\rho}}{\lambda_{\rho}}, \vec{\lambda}_{o, e}=\vec{I} \times \vec{\lambda}_{o, e}$ and $\vec{\lambda}_{o, e}=\hat{x} \lambda_{x}+\hat{y} \lambda_{y}-\hat{z} \lambda_{z o, e} \operatorname{sgn}\left(z-z^{\prime}\right)$. A careful examination of (9) reveals the ordinary contribution is $T E^{z}$ and the extraordinary term (upon later examination of the magnetic field) is $T M^{z}$.

Similar to the steps used in obtaining the forced wave equation in (6), the $\vec{\lambda}_{\rho}$ - domain reflected wave equation with assumed solution form $\overrightarrow{\widetilde{E}}^{r}\left(\vec{\lambda}_{\rho}, z\right)=\overrightarrow{\widetilde{E}}_{0}^{r}\left(\vec{\lambda}_{\rho}\right) e^{j \lambda_{z} z}$ (based on intuition gained via the forced response) is found to be

$$
\left(\overrightarrow{\widetilde{\nabla}} \cdot \vec{\nabla}-\vec{k}^{2}\right) \cdot \overrightarrow{\tilde{E}}^{r}\left(\vec{\lambda}_{\rho}, z\right)=\left(-\vec{\lambda} \cdot \vec{\lambda}-\vec{k}^{2}\right) \cdot \overrightarrow{\tilde{E}}^{r}\left(\vec{\lambda}_{\rho}, z\right)=\vec{W}_{E} \cdot \overrightarrow{\tilde{E}}^{r}\left(\vec{\lambda}_{\rho}, z\right)=0
$$

The non-trivial reflected field is determined by finding the roots of $\operatorname{det} \overleftrightarrow{W}_{E}=0$ and subsequent corresponding eigenvectors, resulting in the ordinary and extraordinary up/down going waves (after some algebraic effort)

$$
\begin{gathered}
\overrightarrow{\tilde{E}}^{r}=\overrightarrow{\tilde{E}}^{r o}+\overrightarrow{\tilde{E}}^{r e}=\overrightarrow{\tilde{E}}_{0}^{r o+} e^{-j \lambda_{z o} z}+\overrightarrow{\tilde{E}}_{0}^{r o-} e^{j \lambda_{z o} z}+\overrightarrow{\tilde{E}}_{0}^{r e+} e^{-j \lambda_{z e} z}+\overrightarrow{\tilde{E}}_{0}^{r e-} e^{j \lambda_{z e} z} \\
\overrightarrow{\tilde{E}}_{0}^{r o \pm}=\left(\hat{x}-\hat{y} \frac{\lambda_{x}}{\lambda_{y}}\right) \tilde{E}_{0 x}^{r o \pm}, \overrightarrow{\tilde{E}}_{0}^{r e \pm}=\left(\hat{x}+\hat{y} \frac{\lambda_{y}}{\lambda_{x}} \pm \frac{\varepsilon_{t}}{\varepsilon_{n}} \frac{\lambda_{\rho}^{2}}{\lambda_{x} \lambda_{z e}}\right) \tilde{E}_{0 x}^{r e \pm}
\end{gathered}
$$

It is interesting to note that these relations are consistent with Gauss's law and that the $y$ and $z$ reflected field components are in terms of the $x$ reflected field component, thus there are four undetermined coefficients $\tilde{E}_{0 x}^{r o+}, \tilde{E}_{0 x}^{r o-}$ and $\tilde{E}_{0 x}^{r e+}, \tilde{E}_{0 x}^{r e-}$ that may be found via the four boundary condition relations on the total fields, namely $\tilde{E}_{x}(z=0)=0$, $\tilde{E}_{y}(z=0)=0, \tilde{E}_{x}(z=d)=0$ and $\tilde{E}_{y}(z=d)=0$. Subsequent boundary condition enforcement leads to the desired reflected electric field dyadic Green's function result (after considerable algebraic effort)

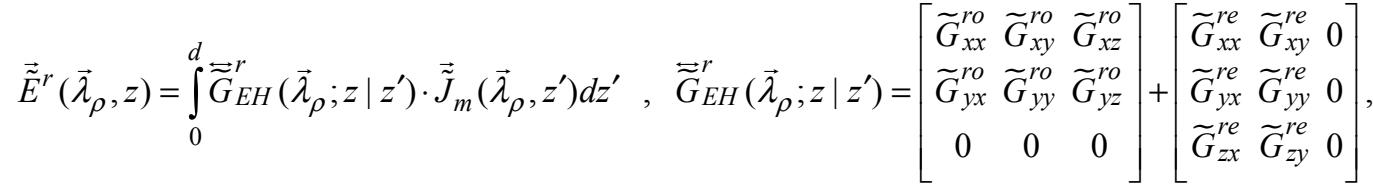

$$
\begin{aligned}
& \widetilde{G}_{y \alpha, E H}^{r o}=-\frac{\lambda_{x}}{\lambda_{y}} \widetilde{G}_{x \alpha, E H}^{r o}=-\frac{\lambda_{x}}{\lambda_{y}} \frac{\lambda_{\alpha} \lambda_{y} \lambda_{z o}\left[e^{-j \lambda_{z o}\left(z+z^{\prime}\right)}+e^{-j \lambda_{z o}\left(2 d+z-z^{\prime}\right)}-e^{-j \lambda_{z o}\left(2 d-z+z^{\prime}\right)}-e^{-j \lambda_{z o}\left(2 d-z-z^{\prime}\right)}\right]}{-2 \lambda_{z o} \lambda_{\rho}^{2}\left(1-e^{-j \lambda_{z o} 2 d}\right)} \ldots \alpha=x, y \\
& \widetilde{G}_{y z, E H}^{r o}=-\frac{\lambda_{x}}{\lambda_{y}} \widetilde{G}_{x z, E H}^{r o}=-\frac{\lambda_{x}}{\lambda_{y}} \frac{\lambda_{y} \lambda_{\rho}^{2}\left[-e^{-j \lambda_{z o}\left(z+z^{\prime}\right)}+e^{-j \lambda_{z o}\left(2 d+z-z^{\prime}\right)}+e^{-j \lambda_{z o}\left(2 d-z+z^{\prime}\right)}-e^{-j \lambda_{z o}\left(2 d-z-z^{\prime}\right)}\right]}{-2 \lambda_{z o} \lambda_{\rho}^{2}\left(1-e^{-j \lambda_{z o} 2 d}\right)}
\end{aligned}
$$

Analogous relations hold for the extraordinary contribution but are omitted for the sake of brevity. Note, the general form of this dyadic Green's function is anticipated due to the inherent $T E^{z}$ and $T M^{z}$ field decomposition. The terms in the numerator of (13) physically represent various wave reflections from the PEC boundaries. It is also important to note that ordinary and extraordinary waves do not couple at the PEC boundaries (as is evidenced by the lack of $\lambda_{z e}$ terms in (13), for example). Next, the magnetic field dyadic Green's function is derived based on the above result.

\section{Magnetic Field Dyadic Green's Function}

The $\vec{\lambda}_{\rho}$ - domain reflected magnetic field dyadic Green's function may be found via (2) and (12), namely

$$
\overrightarrow{\tilde{H}}^{r}\left(\vec{\lambda}_{\rho}, z\right)=-\frac{1}{j \omega \mu_{0}} \overrightarrow{\widetilde{\nabla}} \cdot \overrightarrow{\tilde{E}}^{r}\left(\vec{\lambda}_{\rho}, z\right)=\int_{0}^{d}-\frac{1}{j \omega \mu_{0}} \vec{\nabla} \cdot \overrightarrow{\widetilde{G}}_{E H}^{r}\left(\vec{\lambda}_{\rho} ; z \mid z^{\prime}\right) \cdot \overrightarrow{\tilde{J}}_{m}\left(\vec{\lambda}_{\rho}, z^{\prime}\right) d z^{\prime} \Rightarrow \overrightarrow{\widetilde{G}}_{H H}^{r}=-\frac{1}{j \omega \mu_{0}} \overrightarrow{\widetilde{\nabla}} \cdot \overrightarrow{\widetilde{G}}_{E H}^{r}
$$


Note, the $\vec{\nabla}$ and integral operator interchange is allowed here for the reflected wave since the source point singularity only resides in the principal field. Thus, the reflected magnetic field dyadic Green's function can be readily found via the reflected electric field dyadic Green's function using the simple relation in (14), the details of which are omitted for conciseness.

The principal magnetic field dyadic Green's function derivation must be handled very carefully due to the source point singularity (manifested via the $\left|z-z^{\prime}\right|$ term). Using (2), (8)-(9) and properties of the Dirac delta function, the principal magnetic field may be computed using Faraday's law, namely

$$
\overrightarrow{\tilde{H}}^{p}\left(\vec{\lambda}_{\rho}, z\right)=-\frac{1}{j \omega \mu_{0}}\left[\overrightarrow{\widetilde{\nabla}} \cdot \int_{0}^{d} \overrightarrow{\widetilde{G}}_{E H}^{p}\left(\vec{\lambda}_{\rho} ; z-z^{\prime}\right) \cdot \overrightarrow{\tilde{J}}_{m}\left(\vec{\lambda}_{\rho}, z^{\prime}\right) d z^{\prime}+\int_{0}^{d} \vec{I} \delta\left(z-z^{\prime}\right) \cdot \overrightarrow{\tilde{J}}_{m}\left(\vec{\lambda}_{\rho}, z^{\prime}\right) d z^{\prime}\right]
$$

Due to the presence of the $\left|z-z^{\prime}\right|$ term, the integral must be evaluated in two intervals $[0, z)$ and $(z, d]$ (rigorously, in the principle value sense). Due to the limits of integration being a function of $z$, Leibnitz's rule must be applied during the $\vec{\nabla}$ and integral operator interchange, producing the desired result valid externally and internally to the source region

$$
\begin{aligned}
& \overrightarrow{\widetilde{\nabla}} \cdot \int_{0}^{d} \overrightarrow{\widetilde{G}}_{E H}^{p}\left(\vec{\lambda}_{\rho} ; z-z^{\prime}\right) \cdot \overrightarrow{\tilde{J}}_{m}\left(\vec{\lambda}_{\rho}, z^{\prime}\right) d z^{\prime}=\int_{0}^{d} \overrightarrow{\widetilde{\nabla}}_{0} \cdot \overrightarrow{\widetilde{G}}_{E H}^{p}\left(\vec{\lambda}_{\rho} ; z-z^{\prime}\right) \cdot \overrightarrow{\tilde{J}}_{m}\left(\vec{\lambda}_{\rho}, z^{\prime}\right) d z^{\prime}-(\hat{x} \hat{x}+\hat{y} \hat{y}) \cdot \overrightarrow{\tilde{J}}_{m}\left(\vec{\lambda}_{\rho}, z\right) \\
\therefore & \overrightarrow{\widetilde{G}}_{H H}^{p}\left(\vec{\lambda}_{\rho} ; z-z^{\prime}\right)=-\frac{1}{j \omega \mu_{0}}\left[\vec{\nabla} \cdot \overrightarrow{\widetilde{G}}_{E H}^{p}\left(\vec{\lambda}_{\rho} ; z-z^{\prime}\right)+\hat{z} \hat{z} \delta\left(z-z^{\prime}\right)\right] \quad, \quad \vec{L}=-\hat{z} \hat{z} \frac{\delta\left(z-z^{\prime}\right)}{j \omega \mu_{0}} \ldots \text { depolarizing dyad }
\end{aligned}
$$

As a final note, the various Green's function relations can be computed in the $\vec{\rho}, z$ spatial domain with the aid of (3).

\section{Conclusion}

The principal and reflected electric and magnetic field dyadic Green's functions for a magnetically-excited PEC parallel-plate waveguide filled with a uniaxial dielectric are derived and discussed physically. It is shown that the source maintains a combination of $T E^{z}$ and $T M^{z}$ field sets that do not couple at the PEC boundaries. A relatively simple formulation for computing the magnetic field Green's function is developed. A Leibnitz rule methodology for computing the depolarizing dyad is also presented, resulting in Green's functions that are valid both outside and inside the source region. Future research will involve the use of these Green's functions in characterizing uniaxial materials.

\section{References}

1. A. Nicolson and G., "Measurement of the Intrinsic Properties of Materials by Time Domain Techniques," IEEE Trans. on Instrum. and Meas., 19(4):377-382, Nov. 1970.

2. W. Weir, "Automatic Measurement of Complex Dielectric Constant and Permeability at Microwave Frequencies," Proceedings of the IEEE, 62(1):33-36, Jan. 1974.

3. J. Baker-Jarvis, M. Janezic, P. Domich, and R. Geyer, "Analysis of an Open-Ended Coaxial Probe with Lift-Off for Nondestructive Testing,” IEEE Trans. on Instrum. and Meas., 43(5):711-718, Oct. 1994.

4. M. Ghasr and R. Zoughi, "Multimodal Solution for a Rectangular Waveguide Radiating into a Multilayered Dielectric Structure and its Application for Dielectric Property and Thickness Evaluation," IEEE Instrum. and Meas. Tech. Conf. Proceedings, 2008, pp. $552-556$.

5. M. Hyde and M. Havrilla, "A Nondestructive Technique for Determining Complex Permittivity and Permeability of Magnetic Sheet Materials using Two Flanged Rectangular Waveguides," Progress in Electro. Res., 79:367-386, 2008.

6. H. Chen, Theory of Electromagnetic Waves, McGraw Hill, 1983, pg. 21.

The views expressed in this paper are those of the author and do not reflect the official policy or position of the U.S. Air Force, the Department of Defense, or the U.S. Government. 